\title{
Case report: An adult presenting with congenital radio-ulnar synostosis
}

Gunasekara $\mathrm{KS}^{1}$, Chinthaka $\mathrm{RG}^{1}$, Dissanayake $\mathrm{S}^{2}$

${ }^{I}$ Rheumatology and Rehabilitation unit, District General Hospital, Hambantota

${ }^{2}$ Department of Anatomy, Faculty of Medical Sciences, University of Sri

Jayewardenepura.

\section{Introduction}

Congenital radioulnar synostosis is a rare condition with multi factorial aetiology due to developmental abnormality of upper limbs during early gestation. It results in abnormal osseous fusion between proximal radius and ulna bones leading to fixed pronation deformity of the forearm. This can cause great difficulty in daily activities depending on the extent of the deformity. Congenital radioulnar synostosis is diagnosed during childhood, when abnormal positioning of the forearm is noticed by the parents or teachers. Rarely, this could go undiagnosed till adulthood. Most of these adult patients are diagnosed incidentally when they present to the health care provider for a different reason. The possible reasons for delay in presentation is because this condition is painless and the patient learns to compensate for the deformity using the wrist and the shoulder joints.

Congenital radioulnar synostosis is diagnosed by $\mathrm{x}$ ray of the elbow joint and forearm. Corrective surgery is the treatment of choice when severe limitations of hand functions occur especially affecting day to day essential activities in children. Adults often managed conservatively as they have already adjusted to live with minimal disability. Here, we are reporting a female patient diagnosed with congenital radioulnar synostosis presenting with features of rheumatoid arthritis.

\section{Case report}

A 32 yr old mother of two children presented to the Rheumatology unit, District General Hospital - Hambantota with multiple joint pain for 3 months' duration. The symptoms were gradual in onset involving bilateral elbow, wrist and small joints of the hands. The pain was associated with early morning stiffness lasting more than thirty minutes. The shoulder joint and the lower limb joints were not affected. She has also noted intermittent swelling of the small joints of the hand and the wrist joints. No features such as skin rash to suggest connective tissue disorder. There was no history of trauma. She did not have any significant medical or surgical history nor she had any episodes similar to the current presentation. On examination she had clinical features of active synovitis involving the metacarpophalangeal, proximal interphalangeal and bilateral wrist joints. Her movements of these joints were restricted due to pain hence, unable to make a fist or flex the wrist. 
In addition, she had a significant reduction in supination and pronation at the proximal radioulnar joint. She kept her both forearms in fixed pronated position and further inquiry revealed that she is used to keep her forearms in this position since she could remember. Further to, she has not had any effect on her normal day to day life with this restriction in pronation and supination. Investigations revealed high inflammatory markers with elevated rheumatoid factor titers. Full blood count, thyroid functions and the bone profile were normal. Considering the history, examination and investigations, she was diagnosed to be having sero $(+)$ rheumatoid arthritis and started on Methotrexate and Hydroxychloroquin. $\mathrm{X}$ ray of bilateral elbow joints (Figure $1,2,3)$ were done to investigate for her long term forearm disability and it revealed bilateral radioulnar synostosis of congenital origin. Even though this is unrelated to her present diagnosis of rheumatoid arthritis, we arranged for occupational therapy with activity modification to aim of improving her upper limb functions.

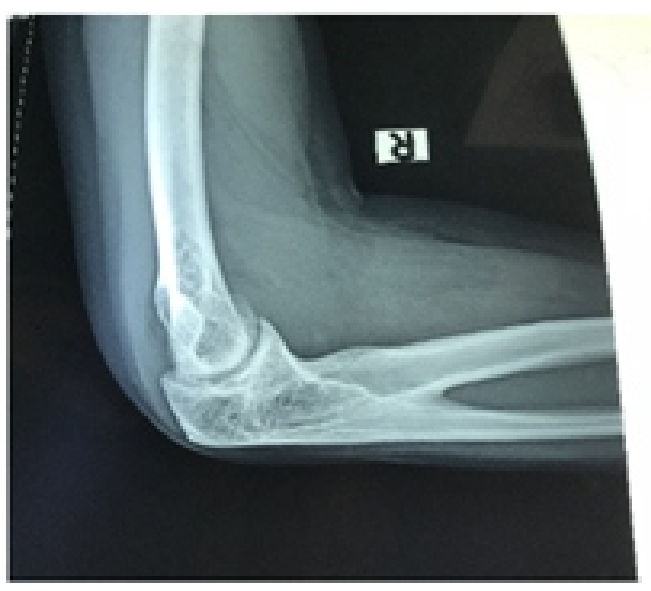

Figure 1: Lateral $\mathrm{x}$ ray of right elbow joint with abnormal articulation between proximal radius and ulna.

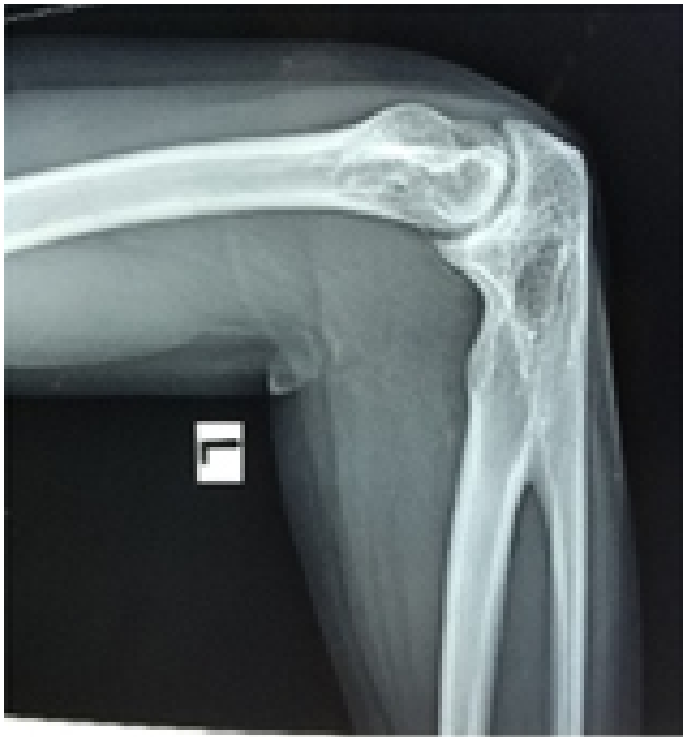

Figure 2: Lateral $\mathrm{x}$ ray of left elbow joint with abnormal articulation between proximal radius and ulna.

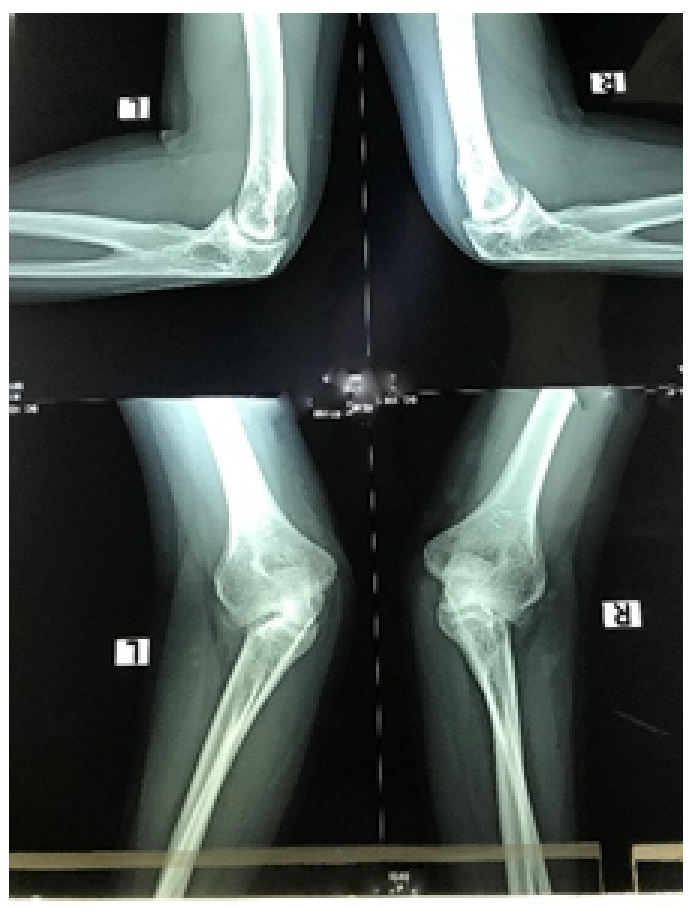

Figure 3: $\mathrm{X}$ rays of bilateral elbow joints 


\section{Discussion}

The forearm consists of two long bones namely radius and ulna. Proximal radial head articulates with the ulna's radial notch to form the superior radio ulna joint. Distally the head of the ulna articulates with the medial side of the distal end of radius to form the inferior radioulnar joint. In between these two joints the two bones are connected with each other by a fibrous interosseous ligament. The main movements at these joints are pronation and supination, where the radial head rotates against the ulna's radial notch in the superior radioulnar joint. This allows radial shaft to rotate around the relatively fixed ulnar shaft. During pronation and supination about 140-180 degrees of rotation occurs at the radioulnar joints which results in rotatory movements of the forearm.

In radioulnar synostosis there is osseous fusion (bony bridge) between the proximal radius and ulna. Radioulnar synostosis can be divided in to two types.

- Congenital radioulnar synostosis

- Post traumatic radioulnar synostosis

The radioulnar synostosis affects supination and pronation of the forearm with minimal impact on the extension and flexion of the forearm at the elbow joint. Post traumatic radioulnar synostosis is a rare complication after forearm or elbow injury that can result in loss of movement and disability. (2) In contrast congenital radioulnar synostosis is a rare condition which result from failure of the normal embryological development of upper limbs. Radius and ulna develop from a common cartilaginous anlage which separates by six weeks of gestation. The failure of this longitudinal segmentation between radius and ulna results in a persistent bridge of tissue (3) which will ossify in to an osseous synostosis. Some authors have reported that there is genetic basis with autosomal dominance inheritance for congenital radioulnar synostosis; associated with chromosomal abnormalities such as duplication of sex chromosomes; congenital syndromes such as Klienfelter's syndrome. (4) Radioulnar synostosis is frequently bilateral and more commonly seen in male patients. (5)

A person with congenital radioulnar synostosis can remain unnoticed as it is asymptomatic and painless. During childhood it can be detected by the health professionals, parents or teachers. Normally the forearm is fixed in an average position of 300 of pronation but with time a person with this condition learns to compensate for the elbow restriction by other movements such as shoulder abduction and adduction. Most of the time elbow flexion is preserved so a person with radioulnar synostosis may ignore his or her limited movements of the forearms until it is detected accidently like in our patient. Once radioulnar synostosis is suspected it can be diagnosed by performing antero-posterior and lateral $x$ rays of the forearm and elbow joint.

Radiographically, there are four types of congenital radioulnar synostosis. 
1. Type I - Synostosis with no osseous bridge. Size of the radial head is reduced.

2. Type II - Synostosis has visible osseous bridge with otherwise normal anatomy.

3. Type III - Long osseous synostosis with hypoplastic and posteriorly dislocated radial head.

4. Type IV - Short osseous synostosis with anteriorly dislocated and mushroom shaped radial head.

Once diagnosed management of congenital radioulnar synostosis depends on several factors including the degree of deformity, impact of the deformity on daily activities and patient's expectations. When this is diagnosed during adulthood; the patient is asymptomatic and leading a normal life with minimal impact on day to day activities, patients tend to opt for conservative management.

Conservative treatment includes occupational therapy focusing on activity modification. Corrective surgery is the other option for patients who have bilateral radioulnar synostosis with very limited forearm movements. Especially if the patient has great difficulty in performing activities such as washing, bathing, brushing to maintain good hygiene, occupation and recreational activities. Surgery is best performed in early adulthood and it is not recommended for older patients with mild deformity.

The patient discussed above had bilateral congenital radioulnar synostosis. However, she has learnt to compensate for the limited range in movements by using her wrists and shoulders. Hence, we educated her regarding the condition and explained that it does not relate to her recent diagnosis of rheumatoid arthritis. She opted for conservative management with counselling, activity modification and occupational therapy.

\section{Conflict of interest}

None declared.

\section{Correspondence}

Dr. Kusala S Gunasekara, Consultant in Rheumatology and Rehabilitation, DGH - Hambantota.

E-mail: kusalagunasekara@yahoo.com Tel: +94 772920748

\section{References}

1. L. Solomon, D. warwick, S. Nayagam, A.G. Apley. Apley's system of orthopedics and fractures $\left(9^{\text {th }}\right.$ ed. $)$,Hodder Arnold,London(2010).p974.

2. Dohn P,Khiami F, Rolland E,Goubier JN.Adult post traumaticsynostosis. Orthop Traumatol surg Res.2012;98(6):709-714.

3. Mital M.A.,Congenital radioulnar synostosis and congenital dislocation of the radial 
Gunasekara KS, Chinthaka RG, Dissanayake S - Case report: An adult presenting with congenital radio-ulnar synostosis

head.Orthop.Clin.North

America.7:375-383.1976.

4. Simons B.P.,Southmayd W.W.,Riseborough E.JJ.Congenital radioulnar synostosis,J.Hand Surg.8:829-838,1983.
5. J.E.Cleary,G.E.Omer Jr; Congenital proximal radioulnar synostosis;Natural history and functional assessment;JBJS, 198 